\title{
Optimization Schemes for the Reversible Discrete Volume Polyhedrization Using Marching Cubes Simplification
}

\author{
David Coeurjolly, Florent Dupont, Laurent Jospin, and Isabelle Sivignon \\ Laboratoire LIRIS/ UMR CNRS 5205 - Université Claude Bernard Lyon 1 \\ Bâtiment Nautibus - 8, boulevard Niels Bohr \\ F-69622 Villeurbanne cedex, France \\ \{david.coeurjolly, florent.dupont, isabelle.sivignon\}@liris.cnrs.fr
}

\begin{abstract}
The aim of this article is to present a reversible and topologically correct construction of a polyhedron from a binary object. The proposed algorithm is based on a Marching Cubes (MC) surface, a digital plane segmentation of the binary object surface and an optimization step to simplify the MC surface using the segmentation information.
\end{abstract}

\section{Introduction}

3D discrete volumes are more and more used especially in the medical area since they result from MRI and CT scanners for example. As 2D images are composed of pixels, these 3D images are composed of voxels. This structure induces many difficulties in the exploitation and study of these objects: for each voxel a value is stored, thus the volume of data for an image is huge which is a problem to get a fluent interactive visualization ; the facet structure (voxels' faces) of the discrete object induces many problems to get a nice visualization that is necessary for medicines, as no rendering nor texture algorithm can be applied. The general idea to solve these problems is to transform discrete volumes into polyhedra with vertices in $\mathbb{R}^{3}$. An important property that must fulfill the Euclidean polyhedron is its reversibility up to a given digitization process (e.g. the result of the digitization must be the original discrete volume itself). In other words, no information are neither created nor lost during the transformation.

Many research activities have already been achieved to find solutions to compute this reversible transformation, using Euclidean geometry or discrete geometry 1223456 . To get a good visualization of discrete volumes, classical methods use the Marching Cubes algorithm 78, which considers local voxel configurations to replace them by small triangles. Even if these methods offer a good visualization, it does not provide a good data compression (huge number of facets) but we have a first reversible solution. Digital geometry solutions deal with a first step that segments the object boundary into pieces of digital plane 92101113 . The digital plane is a fundamental object for this problem because reversibility properties exist. The next step consists in associating a polygon to each piece of digital plane and finally to construct the Euclidean 
polyhedron while sewing the polygons. The major problem of these methods is to ensure both the reversibility and the correct topology of the polyhedron.

In 4, we have proposed a polyhedrization algorithm with the following properties: it computes a reverse polyhedrization of the input digital object with the warranty that the obtained polyhedron is topologically correct. More precisely, the final polyhedron is a combinatorial 2-manifold. This algorithm is based on a simplification of the Marching-Cubes surface with digital plane segmentation information. In the following, we extend this algorithm using linear programming techniques to reduce the number of facets of the final object while preserving both the reversibility of the surface and its topology.

In section 2, we describe the preliminaries with a review of existing algorithms. In section 3 , we detail the Marching-Cubes based simplification algorithm and its optimizations to obtain a polyhedron from a discrete object with a reduced number of facets.

\section{Preliminaries}

\subsection{The Marching-Cubes Algorithm}

Let us assume a discrete 3D image that maps a value $V(x, y, z) \in \mathbb{R}$ to each grid point $(x, y, z) \in \mathbb{Z}^{3}$. The image $V$ can also be considered as a density function on a subset of $\mathbb{Z}^{3}$. The Marching-Cubes (MC) algorithm was first introduced by Lorensen and Cline [7] to extract a triangulated surface from $V$ corresponding to an iso-density value. The first application of this work was the visualization of iso-density surfaces in medical imaging. We first consider cubic cells of coordinate $(x, y, z)$ whose vertices are placed on the 8 input samples $(x+i, y+j, z+k)$ of the volume data, with $i, j, k \in\{0,1\}$. The triangulated iso-surface given by the Marching-Cubes algorithm is locally computed according to the way the surface intersects each cell of $V$ using a look-up table with 14 possible configurations (see Figure11). The coordinates of the MC vertices along an edge of a cell is given by an interpolation process between the values of $V$ and the chosen iso-level.

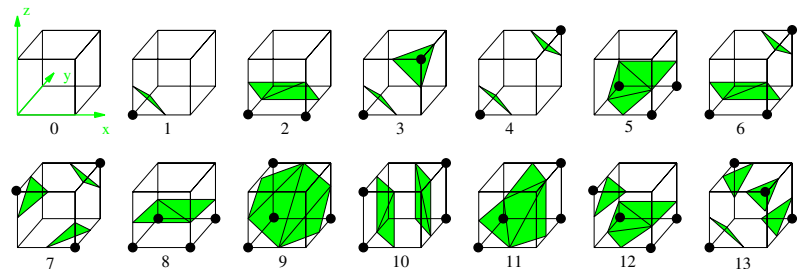

Fig. 1. The 14 different standard triangulations of the Marching-Cubes algorithm

Note that some of original Lorensen and Cline's configurations may lead to ambiguities in the reconstruction and thus construct surfaces with holes. To have 
properties on the topology of the reconstruction, we need a process that disambiguates the configurations according to the topology of the input discrete surface. The configurations presented in the Figure 1 correspond to a $(18,6)$-surface [812. Hence, if the binary object is 6-connected, the triangulated surface is a combinatorial 2-manifold, i.e. closed, oriented and without self crossing [812]. In the following, we consider the Object Boundary Quantization (OBQ) scheme, also called Gauss digitization [13] : given an region $P \subset \mathbb{R}^{3}$ in $\mathbb{R}^{3}$, the OBQ digitization of $P$ is the set of voxels $P \cap \mathbb{Z}^{3}$. If a binary object is considered, i.e. if $V(x, y, z) \in\{0,1\}$, for all $x, y$ and $z$, from [4, we have the following lemma (see Figure 2):

Lemma 1 ([4]). The Marching-Cubes surface of a digital object, obtained with a an iso-level in ]0, 1 [, is a reversible polyhedrization of the binary object according to the Object Boundary Quantization model.
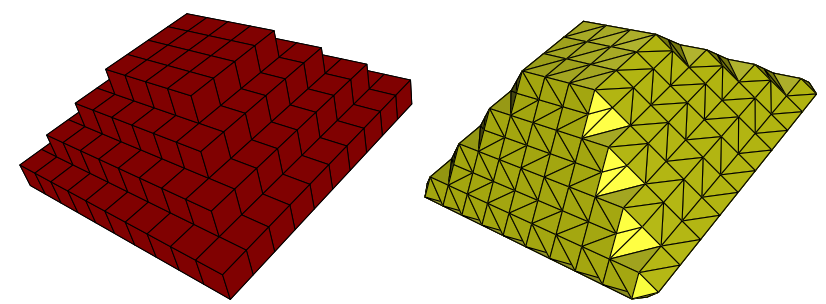

Fig. 2. A binary 3D object and the obtained Marching-Cubes surface

Given a discrete object, the surfels are cellular elements of the unit cube and are defined as the square shared by a voxel $p$ in the binary object and a voxel $q$ in its complementary, denoted $\{p, q\}$. Hence, according to the MC configurations, we have a one-to-one and onto mapping between $\mathrm{MC}$ vertices and surfels of the binary object. Indeed, vertices of the MC surface belongs to the ] $p q[$ straight line segment (the vertex cannot be neither $p$ nor $q$ ). Furthermore, it is easy to see that moving a $\mathrm{MC}$ vertex along its $] p q$ [ intervals do not change the result of Lemma 1 .

In the following, we propose a reversible polyhedrization based on a simplification of the Marching-Cubes surface.

\subsection{Digital Plane Segmentation of a Discrete Surface}

In order to simplify the Marching-Cubes surface, we compute a decomposition of the digital surface into coplanar set of surface elements. Consider a set of voxels $\mathcal{V}$, this set is a piece of digital plane with $x \geq z, y \geq z$ and $z>0$ if and only if there exists a Euclidean plane containing $\mathcal{V}$ in its digitization. In other words, there exists $(\alpha, \beta, \gamma)$ in $[0,1]^{2} \times[0,1[$ such that $\mathcal{V}$ is included in $P=\left\{(x, y, z) \in \mathbb{Z}^{3} \mid 0 \leq \alpha x+\beta y+\gamma+z<1\right\}[2[14[13]$. Thus we can define the preimage of $\mathcal{V}$ as the set of $(\alpha, \beta, \gamma)$ parameters fulfilling this condition 10314]. In the following, we call digital plane segments (DPS) coplanar sets of voxels. 
This preimage is an efficient tool for the recognition process: given a set of voxels $\mathcal{V}$, decide if $\mathcal{V}$ is a DPS and if so, compute its parameters [10315].

The definition given previously assumes that a direction is chosen before the recognition process. Generalizing the directional constraint, each direction of the set $D=\{(1,0,0),(-1,0,0),(0,1,0),(0,-1,0),(0,0,1),(0,0,-1)\}$ defines a preimage associated to a given set of voxels. In most cases, only one of those preimages is not empty for a given set of voxels. Considering a direction $\boldsymbol{d} \in D$ and according to the preimage definition, the preimage is the set of Euclidean planes crossing all the segments $[p q$ [ where $p$ is a voxel of $\mathcal{V}$ and $q$ is the voxel of coordinates $p+\boldsymbol{d}$. Note that in practice, $p$ is a voxel of the object while $q$ belongs to the background.

The decomposition algorithm we use is the one presented in 4, which consists in labeling every surfel of the object's surface such that the following property is fulfilled:

Lemma 2 ([4]). Consider a surface surfel $s$ defined by the two voxels $p$ and $q=p+d, d \in D$. If $s$ is labeled with $P$, then all the Euclidean planes of the preimage of $P$ in direction $d$ cross the segment $[p q[$.

This property is of major importance for our problem. Indeed, let $(\alpha, \beta, \gamma)$ be an Euclidean plane of the preimage associated to $s$. According to this property, it crosses the segment $[p q$ [ at a point $r$. If we move $v$ to $r$, i.e., if we project $v$ onto $(\alpha, \beta, \gamma)$ in the $\boldsymbol{p} \boldsymbol{q}$ direction, we do not change the digitization of $v$. Note that it is straightforward to consider intervals $] p q$ [ instead of intervals $[p q[$, we just have to handle strict inequalities in the digital plane definition without changing the algorithms.

\section{Marching-Cubes Simplification and Optimization}

Since there is a one-to-one and onto mapping between the MC vertices and the surfels of the input discrete object, we introduce a label on MC triangles as follows:

Definition 1 (Homogeneous and non-homogeneous triangle). Let $T$ be a triangle of the MC surface, $T$ is homogeneous (H) if its three vertices are associated to surfels belonging to the same digital plane. Otherwise, $T$ is called non-homogeneous (NH). If $T$ is homogeneous, $T$ is labeled with the digital plane segment label of its vertices.

Furthermore, we can define the 2-NH triangle (resp. 3-NH triangle) if the number of distinct discrete plane segments associated to its vertices is exactly 2 (resp. 3).

In the following, we introduce a projection process of a $\mathrm{MC}$ vertex onto an Euclidean plane: let $v$ be a MC vertex and $p, q$ be the two voxels ( $p$ belongs to the object and $q$ to the background) such that $v$ is associated to the surfel $\{p, q\}$. Thus, only the projection of $v$ onto an Euclidean plane $P$ according to the $\boldsymbol{p} \boldsymbol{q}$ direction is considered. 


\subsection{Homogeneous Triangles Case}

Using 4, we have the following result on H-triangles:

Lemma 3 ([4]). Let $v$ be a vertex of an $H$-triangle, let $P$ be an Euclidean plane from the preimage of the discrete plane associated to the triangle. The projection of $v$ onto $P$ does not change neither the reversibility nor its topological properties of the global surface.

This lemma can easily be proven by definition and properties of the discrete plane segmentation process and using Lemma 2

In [4, the authors design a simplification algorithm based on the previous lemma to remove the homogeneous triangles: let $S$ be a connected set of $\mathrm{H}$ triangle with the same label, they extract from the DPS preimage associated to $S$ an Euclidean plane $P$. Then, if we project all vertices of $S$ onto $P$, triangles in $S$ become coplanar. Finally, a post-processing step converts all connected sets of $\mathrm{H}$-triangles with the same label into a single facet. At each step of this algorithm, we ensure the reversibility property and the final surface is still a combinatorial 2-manifold. Note that no assumption is needed during the choice of the plane $P$.

As presented in Figure 8 for each connected set of $\mathrm{H}$-triangle with the same label, we have obtained a facet. NH-triangles allow to sue together all the facets maintaining the topological property of the polyhedron.

In the next section, we present a linear programming framework to extract, from the preimage, an appropriate Euclidean plane $P$ in order to remove NHtriangles.

\subsection{Non-homogeneous Triangles Case}

The basic idea to remove the NH-triangles consists in adding linear constraints in the DPS preimages. Then, the choice of the Euclidean plane $P$ is made by a linear inequality system solver.

However, to have an efficient algorithm, we restrict the problem using the following two heuristics:

Local analysis: let us examine the $2 \mathrm{D}$ reconstruction presented in the Figure 3 If we consider the OBQ scheme, both polygons are correct regarding to the reversibility property. However, the visual aspect of the dashed polygon compared to the initial binary object is worse than the bold one. Hence, our reconstruction is restricted to a polyhedron defined in the cells defined by the MC surface. More precisely, when a modification of an NH triangle is performed, the result must belong to the $\mathrm{MC}$ cell associated to the triangle. This heuristic is a restriction on the possible reconstruction but it allows to design efficient algorithms since the surface properties (reversibility and topology) can be ensure using local analysis. Other arguments justifying this approach are based on the fact that the OBQ digitization scheme associated to $\mathrm{MC}$ surfaces is not a complete digitization model [16.

Linear programming problem in dimension 3: during the DPS recognition process, we have used linear programming algorithms in dimension 3 to 
compute the preimages 10315 . In this optimization process, the dimension of the linear constraint system that conducts the $\mathrm{NH}$ triangle simplification must be bounded by 3. Even if this choice influences and reduces the scope of the algorithm, we limit the computational cost of the linear programming solver this way. Furthermore this process is still consistent with the DPS preimage parameter space.
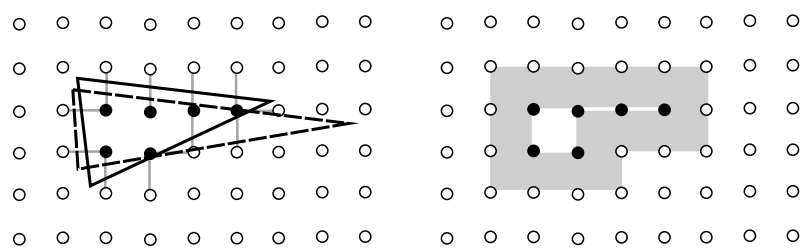

Fig. 3. (left): Two possible polygonalizations of a binary object (dark grey dots). The grey segment represent the ] $p q$ [ intervals in the OBQ scheme. (right): The light grey area define the allowed location of the polygon vertices we use (hence only the bold polygon in the left figure would be considered in our algorithm).

Using these heuristics, the process can be summarized as follows: when a $\mathrm{NH}$ triangle $T$ is considered, two different cases may occur during the simplification process (see Figure 4):

- remove an edge from $T$ : in this case, the edge is collapsed into a point. Furthermore, such a point belongs to a face of the MC cell containing $T$. Hence, a $2 \mathrm{D}$ processing is used to constraint the new point to be in the MC cell (see Figure 5).

- Remove a triangle : the triangle is collapsed into a single point and we have to ensure that the point belongs to the MC cell.

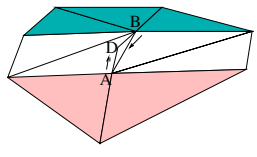

(a)

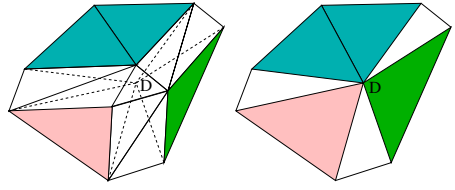

(b)

Fig. 4. Illustration of the removal of an edge $(a)$ and a triangle $(b)$ of the MC surface

Let $T$ be a NH-triangle, to check if an edge of $T$ can be removed, we consider the three $\mathrm{MC}$ cell faces on which $T$ edges are defined (see Figure 5). From the three edges of $T$, at least one out of the three edges of $T$ is such that its vertices do not belong to the same discrete plane segment. Let $\mathcal{P}_{1}$ and $\mathcal{P}_{2}$ be the two preimages associated to such edge $e$. The edge $e$ can be removed if for all $P_{1} \in \mathcal{P}_{1}$ 
and $P_{2} \in \mathcal{P}_{2}$, the intersection of $P_{1}$ and $P_{2}$ belongs to the MC cell face associated to $e$. It is not possible to linearly express those conditions without changing the dimension of the linear programming problem. To solve that point, we consider two approaches to obtain sufficient conditions on the intersection of $P_{1}$ and $P_{2}$.
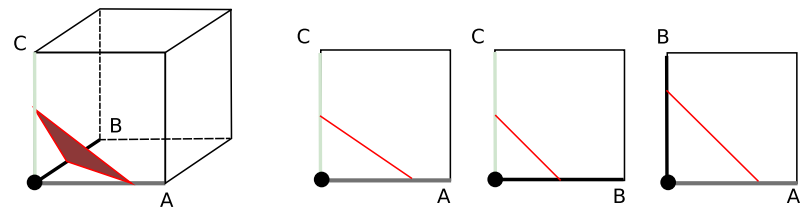

Fig. 5. Illustration of the $2-\mathrm{D}$ decomposition of a $\mathrm{MC}$ cell into its faces in order to decide if an edge of a cell triangle can be removed

Global Simplification. First of all, we have a global simplification process to remove NH elements. In this step, we only consider the simple MC configurations, i.e. the configurations with a single surface patch $(1,2,5,8,9,11$ in the Figure 11). In the other configurations, we have to check the intersection of the two surface patches and we cannot add linear constraints to ensure the topology during the global simplification. The analysis of these configurations is done during the greedy simplification.

To obtain sufficient conditions on the intersection of $P_{1}$ and $P_{2}$, we can list three cases (see Figure 6), depending of how many voxels belong to the object on the considered face of the MC cell. If only one voxel $A$ belongs to the object on a face $A B C D$, then the plane $P_{1}$ associated to the surfel $\{A, B\}$ crosses the segment $C D$ and $P_{2}$ associated to the surfel $\{A, D\}$ crosses the segment $B C$. Thus we ensure that the intersection of $P_{1}$ and $P_{2}$ is inside the square. If we consider the case where two voxels belongs to the object on a face, then there is no interesting linear constraints. If we consider the case where only one voxel $C$ does not belong to the object on a face $A B C D$, then we will have the plane $P_{1}$ associated to surfel $\{D, C\}$ cross the segment $A B$ and the plane $P_{2}$ associated to surfel $\{B, C\}$ cross the segment $A D$. As in the first case, those conditions ensure that the intersection of the two planes is inside the square (see Figure 6). Finally, these constraints lead to simple linear constraints in dimension 3 that reduce both the preimages $\mathcal{P}_{1}$ and $\mathcal{P}_{2}$ to preimages $\mathcal{P}_{1}^{\prime}$ and $\mathcal{P}_{2}^{\prime}$. Hence, if $\mathcal{P}_{1}^{\prime}$ and $\mathcal{P}_{2}^{\prime}$ are not empty, whatever $P_{1} \in \mathcal{P}_{1}^{\prime}$ and $P_{2} \in \mathcal{P}_{2}^{\prime}$, the intersection of $P_{1}$ and $P_{2}$ belongs to the face $A B C D$ of the $\mathrm{MC}$ cell, ensuring the reversibility of the modified surface. If one of the two preimages is empty, the edge is not removed.

Greedy Simplification. This step consists in fixing planes one by one, to have more flexible constraints on the preimage of the remaining planes, and to be able to handle more cases. So the scheme is to fix one Euclidean plane $P_{1}$ (arbitrarily chosen in its associated preimage $\mathcal{P}_{1}$ ). Then, if $T$ is a $\mathrm{NH}$ triangle associated to the DPS represented by the Euclidean plane $P_{1}$ and another DPS with preimage $\mathcal{P}_{2}$, we insert linear constraints on $\mathcal{P}_{2}$ to control the intersection between $P_{1}$ and 

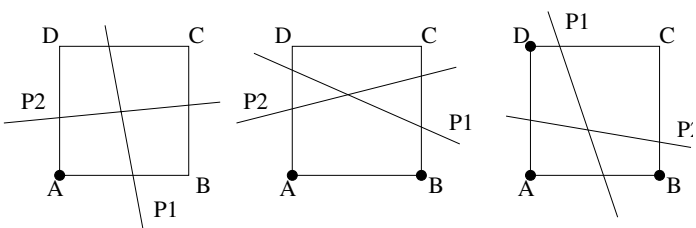

Fig. 6. The three possible cases to define sufficient conditions to remove an edge of a $\mathrm{NH}$ triangle

$P_{2}$. Since $P_{1}$ is fixed, the intersection point being inside the MC cell face is given by linear constraints in the $\mathcal{P}_{2}$ parameter space.

Indeed let us consider a plane $P_{1}$ and a mobile plane $P_{2}$ on a face $A B C D$ (see Figure 7), if $I$ is the intersection of $P_{1}$ and $P_{2}$, to ensure that $I$ is inside the square $A B C D$, we have the constraints:

$$
\left\{\begin{array}{c}
x_{A}<x_{I}<x_{A}+1 \\
y_{A}<y_{I}<y_{A}+1
\end{array}\right.
$$

As $x_{A}$ and $y_{A}$ are constants and $x_{I}, y_{I}$ only depend on the $\mathcal{P}_{2}$ parameters, these inequalities result in linear constraints.
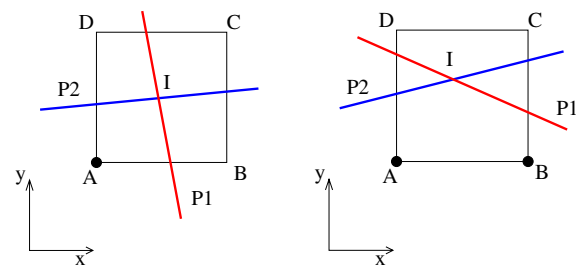

Fig. 7. Illustration of the greedy simplification approach

Finally, if we fix a plane $P_{1}$ for a DPS, we propagate this piece of information to each neighboring DPS preimages. This process is greedy since we do not backtrack on the choice of $P_{1}$. Once all neighboring DPS have been considered, the greedy step can choose another Euclidean plane in another preimage and the process starts over.

Concerning 3-NH triangles, we need to ensure that the intersection of the 3 planes is inside the MC cell associated to the triangle. To do so, we need 2 of the 3 associated planes to be fixed to get linear constraints from the inequalities:

$$
\left\{\begin{array}{l}
x_{A}<x_{I}<x_{A}+1 \\
y_{A}<y_{I}<y_{A}+1 \\
z_{A}<z_{I}<z_{A}+1
\end{array}\right.
$$

Furthermore, we need to constrain the planes such that their intersection two by two with the associated face of the MC cell is inside that face. This leads to the same constraints as in the $2-\mathrm{NH}$ case. 


\subsection{Overall Algorithm}

In this section, we sketch the overall simplification algorithm based on the two approaches presented above.

The first step is to convert the discrete object into a triangular polyhedron, this is done with the Marching Cubes algorithm previously presented. The next step is to segment the discrete object surface into DPS, each of these segments being associated with their preimage. Note that we only consider the NH triangles such that their associated MC configuration is in $(1,2,5,8,9,11)$ (see Figure 1). Indeed, other configurations lead to two or three components of the MC surface and defining sufficient conditions to avoid self-crossings of the surface using constraints in dimension 3 would have led to too restrictive conditions. Hence these triangles are not optimized.

In the first place, we perform a global optimization. We have an unsorted list of all $\mathrm{NH}$ triangles processed one by one. If we have a 2-NH triangle, we can arbitrarily remove any of the two edges since it does not change anything on the final number of facets. The removal consists in adding constraints over the two planes. If a constraint makes one of the preimage empty, then the constraints are removed, and the removal is handled in the next optimization. In this step, we do not handle the 3 -NH because we cannot write linear constraints for a triangle removal.

When all triangles have been processed, we start with the second step of the $\mathrm{NH}$ removal. We arbitrarily choose a $2-\mathrm{NH}$ triangle and fix one of its planes with the barycenter of its preimage. When this is done, we add constraints over the second plane to remove that triangle, if possible. Then we move to an adjacent triangle and repeat the process. If it is a $3-\mathrm{NH}$, we skip it until two out of its planes are fixed. When a triangle cannot be removed and the list of adjacent triangles is empty, we choose a new 2-NH triangle and apply the same process on that one. At the end of the process, all planes have been fixed and we can displace all vertices on the intersection of their euclidean plane and their $] p q[$ segment. Finally, we group coplanar triangles into polygons.

This algorithm can be sketched as follows:

1. Computation of the MC surface

2. Decomposition of the discrete object surface into DPS

3. Optimization on NH triangles, i.e. find an Euclidean plane in each DP preimage:

(a) Step 1: global optimization, processing of all 2-NH triangles

(b) Step 2: greedy optimization, fixing planes one by one to try to remove remaining $\mathrm{NH}$ triangles

4. Vertices displacements and simplification of coplanar triangles.

Lemma 4. The algorithm presented above constructs a reversible polyhedron which is a combinatorial 2-manifold.

Proof. The proof is straightforward according to Property 2 , To prove the topology, since the MC surface is a combinatorial 2-manifold [812] and we can locally 
prove that treatments on both $H$ triangle and $N H$ triangle do not change the topology: no holes are created, no self-crossings are introduced since we remain on the MC cell, and both the orientation and the combinatorial aspects of the surface are maintained. Hence, the final overall surface is still a combinatorial 2-manifold (see 44 for details on the $H$ triangle treatment). Furthermore, since each new element (facets and vertices) belongs to the MC cells in which the surface is defined, the OBQ digitization of the final polyhedron exactly corresponds to the input set of voxels. Note that since the topology is preserved, the polyhedral surface is still oriented and the OBQ digitization scheme is still well defined.

The computational cost of the algorithm is highly dependent on the size of the DPS preimages [15]17. Indeed, a bound on these objects allow us to have computational costs for the DPS decomposition step and for the efficiency of the linear programming solver used to reduce the preimages.

\section{Experiments and Results}

In the experimentation, the digital plane segmentation has been performed using an implementation of the algorithm proposed in [3. The output of this algorithm is a labeling of each surfel with a digital plane segment label, associated to a
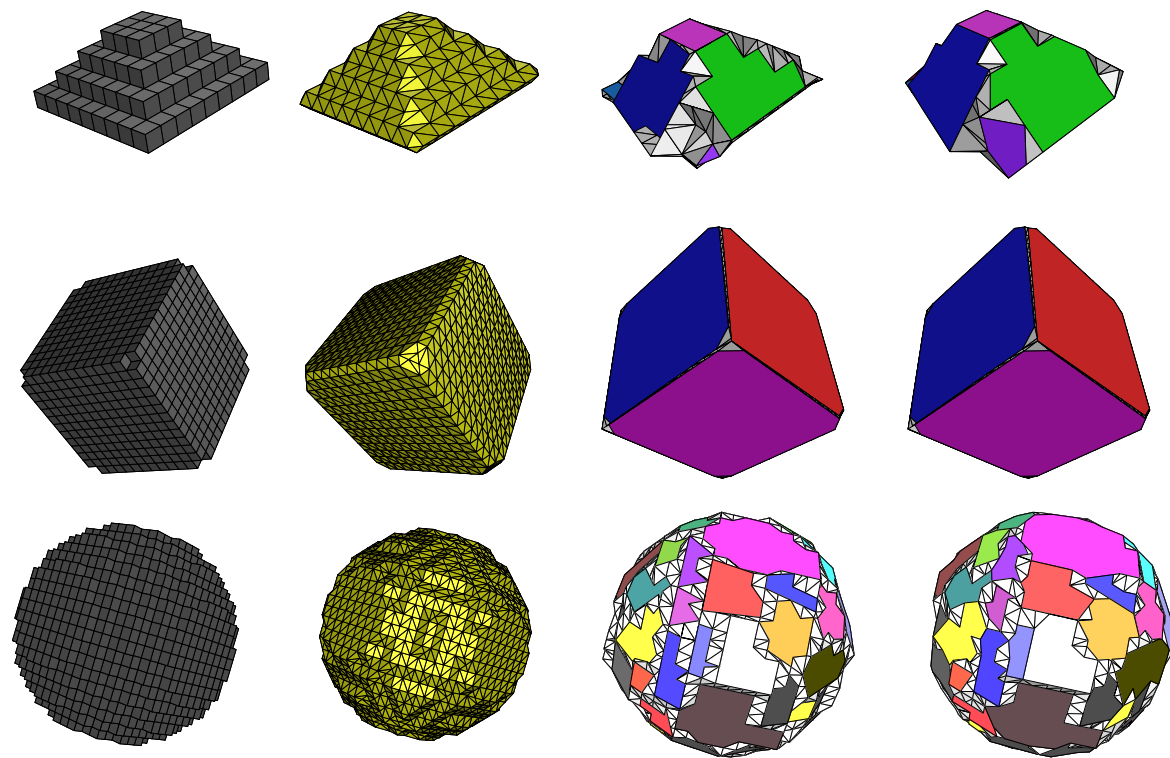

Fig. 8. Comparison between the normal simplification, and the simplification including NH triangles removal. From left to right : facetized voxel representation, MC surface, normal simplfication, and simplification using $\mathrm{NH}$ triangles removal. 
Table 1. Some results of the presented work

\begin{tabular}{l|c|c|c|c|c}
\multirow{2}{*}{ object } & \multicolumn{3}{|c|}{ MC } & \multicolumn{2}{c}{ Removal rate } \\
\cline { 2 - 6 } & \# MC triangles & \# H triangles & \# NH triangles & NH triangles & global \\
\hline pyramid_4 & 512 & 342 & 170 & $62 \%$ & $87 \%$ \\
rd_cube_7 & 2024 & 1720 & 304 & $89 \%$ & $98 \%$ \\
sphere_10 & 3656 & 2200 & 1456 & $37 \%$ & $75 \%$
\end{tabular}

preimage. The modification of the preimages during the NH-triangle simplification have been performed using a linear programming library in dimension 31 .

Figure 8 and Table 1 show some experiments. We can notice that in all presented cases, the global removal rate is always greater than $75 \%$ which also holds for most experimented objects. The NH triangle removal shows an improvement of at least $30 \%$ and up to $60 \%$ compared to the initial algorithm. On the rounded cube, the algorithm could remove almost all triangles keeping only one polygon for each face, one for some edges, and some triangles for corners where the algorithm was not really efficient. On the sphere we see one of the worst result of the algorithm which is still decent, the digital plane recognition showed pretty good result considering we were processing a sphere and the $\mathrm{NH}$ simplification could remove a good part of the remaining triangles.

\section{Conclusion}

In this article, we have presented an algorithm to construct a reversible polyhedron from a digital plane segmentation of a binary object. Once the digital plane segmentation is computed, the proposed algorithm is based on a simplification and an optimization of the Marching-Cubes surface. The next step for this work would be to perform exhaustive comparisons between this algorithm and classical simplification schemes of MC surfaces in the Modeling community according to the number of remaining facets. Note that compared to these algorithms, the reversible property ensured by our technique is an important advantage. Note that to extend this work to handle large volumes, we only have the bottleneck implied by the digital plane decomposition step: to have exact computations, a rational arithmetic must be used to recognize the DPS. When large digital plane segments are considered, the arithmetical size of internal rational numbers quickly increases. Hence, further preliminary analysis on the computational aspects of the DPS recognition are required.

\section{References}

1. Françon, J., Papier, L.: Polyhedrization of the boundary of a voxel object. In: 8th International Workshop in Discrete Geometry for Computer Imagery, SpringerVerlag, LNCS, 1568 (1999) 425-434

${ }^{1}$ http://www.cs.washington.edu/research/constraints/cassowary/ 
2. Debled-Rennesson, I.: Etude et reconnaissance des droites et plans discrets. PhD thesis, Université Louis Pasteur (1995)

3. Sivignon, I., Dupont, F., Chassery, J.M.: Decomposition of a three-dimensional discrete object surface into discrete plane pieces. Algorithmica 38(1) (2004) 25-43

4. Coeurjolly, D., Guillaume, A., Sivignon, I.: Reversible discrete volume polyhedrization using marching cubes simplification. In: SPIE Vision Geometry XII. Volume 5300., San Jose, USA (2004) 1-11

5. Sivignon, I., Dupont, F., Chassery, J.M.: Reversible polygonalization of a 3D planar discrete curve: Application on discrete surfaces. In: International Conference on Discrete Geometry for Computer Imagery. (2005) 347-358

6. Dexet., M.: Design of a topology based geometrical discrete modeler and reconstruction methods in $2 \mathrm{D}$ and $3 \mathrm{D}$. $\mathrm{PhD}$ thesis, Laboratoire SIC, Université de Poitiers (2006) (in French).

7. Lorensen, W.E., Cline, H.E.: Marching cubes: a high resolution 3D surface construction algorithm. In Stone, M.C., ed.: SIGGRAPH '87 Conference Proceedings (Anaheim, CA, July 27-31, 1987), Computer Graphics, Volume 21, Number 4 (1987) 163-170

8. Lachaud, J.O.: Topologically defined isosurfaces. In: 6th Discrete Geometry for Computer Imagery, LNCS 1176, Springer-Verlag (1996) 245-256

9. Borianne, P., Francon, J.: Reversible polyhedrization of discrete volumes. In: 4th Discrete Geometry for Computer Imagery, Grenoble, France (1994) 157-168

10. Vittone, J., Chassery, J.M.: Recognition of digital naive planes and polyhedization. In: Discrete Geometry for Computer Imagery. Number 1953 in LNCS, Springer (2000) 296-307

11. Klette, R., Sun, H.J.: Digital planar segment based polyhedrization for surface area estimation. In Arcelli, C., Cordella, L.P., Sanniti di Baja, G., eds.: International Workshop on Visual Form 4. Volume 2059 of Lect. Notes Comput. Sci., SpringerVerlag (2001) 356-366

12. Lachaud, J.O., Montanvert, A.: Continuous analogs of digital boundaries: A topological approach to iso-surfaces. Graphical Models and Image Processing 62 (2000) 129-164

13. Klette, R., Rosenfeld, A.: Digital Geometry: Geometric Methods for Digital Picture Analysis. Series in Computer Graphics and Geometric Modelin. Morgan Kaufmann (2004)

14. Brimkov, V., Coeurjolly, D., Klette, R.: Digital planarity - a review. Technical Report RR-2004-024, Laboratoire LIRIS, Université Claude Bernard Lyon 1 (2004)

15. Coeurjolly, D., Sivignon, I., Dupont, F., Feschet, F., Chassery, J.M.: On digital plane preimage structure. Discrete Applied Mathematics 151(1-3) (2005) 78-92

16. Andrès, E.: Discrete linear objects in dimension n: the standard model. Graphical models 65(1-3) (2003) 92-111

17. Coeurjolly, D., Brimkov, V.: Computational aspects of digital plane and hyperplane recognition. In: 11th International Workshop on Combinatorial Image Analysis. Volume 4040 of Lecture Notes in Computer Science., Springer-Verlag (2006) 291306 